\title{
Personality Traits, Interpersonal Identity, and Relationship Stability: Longitudinal Linkages in Late Adolescence and Young Adulthood
}

\author{
Theo A. Klimstra $\cdot$ Koen Luyckx $\cdot$ Susan Branje • \\ Eveline Teppers $\cdot$ Luc Goossens $\cdot$ Wim H. J. Meeus
}

Received: 7 September 2012/ Accepted: 6 November 2012/Published online: 13 November 2012

(C) Springer Science+Business Media New York 2012

\begin{abstract}
Adolescence and young adulthood are characterized by important changes in personality, changes toward a more stable identity, and the establishment of intimate relationships. We examined the role of personality traits in establishing intimate relationships, the interplay between personality traits and interpersonal identity processes during these relationships, and the role of interpersonal identity processes and personality traits in the dissolution thereof. For this purpose, we used longitudinal data on 424 female college students (mean age at $\mathrm{T} 1=18.6$ years; Sample 1) and 390 late adolescents drawn from a community sample $(56.7 \%$ female; mean age at $\mathrm{T} 1=19.7$ years; Sample 2). Especially highly extraverted individuals were likely to become involved in a relationship. Neuroticism was associated negatively, and Agreeableness and Conscientiousness were associated positively with a stronger sense of interpersonal identity within intimate relationships. Finally, the importance of interpersonal identity processes was underscored by the fact that these processes, and not so much personality traits, predicted relational breakups. Overall, the present study
\end{abstract}

Theo A. Klimstra, Koen Luyckx are postdoctoral researchers at the Fund for Scientific Research Flanders (FWO).

T. A. Klimstra (凹)

Department of Developmental and Clinical Psychology, Tilburg University, Postbus 90153, 5000 LE Tilburg, The Netherlands e-mail: t.a.klimstra@uvt.nl

T. A. Klimstra · K. Luyckx · E. Teppers - L. Goossens School Psychology and Child and Adolescent Development, Catholic University Leuven, Leuven, Belgium

S. Branje · W. H. J. Meeus

Research Centre Adolescent Development, Utrecht University, Utrecht, The Netherlands provides important insights into the role of personality and identity in the initiation, maintenance, and dissolution of intimate relationships in late adolescence and young adulthood.

Keywords Personality - Identity formation · Relationships · Big five $\cdot$ Adolescence $\cdot$ Longitudinal

\section{Introduction}

There is evidence for remarkable stability in personality traits across the life course. Yet, changes are possible, and such changes seem to be especially prevalent from adolescence to young adulthood (Roberts et al. 2006). Specifically, substantive changes towards maturity in most of the Big Five dimensions have been observed, as levels of Neuroticism tend to decrease, whereas levels of Agreeableness and Conscientiousness tend to increase. High levels on these traits are positively associated with psychological well-being (Kotov et al. 2010). Therefore, it is important to understand what the antecedents and correlates of changes in personality traits are.

One possible correlate of personality maturation is the acquisition of age-graded social roles, such as establishing a career and a family. Although such roles can more or less be fulfilled in early stages of life (e.g., high school students with a strong drive to become surgeons, who therefore put a lot of effort in their education), they are almost forced upon us by society in late adolescence and young adulthood (e.g., Roberts and Wood 2006).

From a social investment perspective, increased identification with such roles is viewed as a driving force behind personality maturation (Lodi-Smith and Roberts 2007). Becoming engaged in a stable intimate relationship is 
among the most prominent of the aforementioned social roles (e.g., Roberts and Wood 2006).

The process of becoming engaged in a stable intimate relationship is two-fold. First, one needs to find an intimate partner. Several studies have shown that personality traits may play a role in this regard, but these studies are marked by important limitations. Second, and most important from a social investment perspective, individuals can identify themselves with this relationship. Identification with social roles such as intimate relationships can be captured with identity processes (e.g., Grotevant et al. 1982; Marcia 1966). The importance of these identity processes is underscored by the fact that they tend to be associated with, for example, elevated levels of depressive symptoms (e.g., Crocetti et al. 2008; Luyckx et al. 2008). Yet, the linkages between personality traits and identity processes are generally understudied, with studies relating these processes in the specific domain of intimate relationships to personality traits being non-existent. The present study also will examine whether identity processes are indeed good indicators of investment in a relationship. That is, if identity processes are good indicators of investment in relationships, these processes should predict the subsistence of intimate relationships (i.e., a directly observable indicator inversely associated with investment) above and beyond stable dispositions such as personality traits. This issue has received little attention in previous studies, but will be considered in the present study. Thus, the purpose of the present study was to examine the role of personality traits and interpersonal identity processes across the course of intimate relationships. For this purpose, we examined the role of personality traits in the emergence of intimate relationships, the interplay between personality traits and interpersonal identity processes during such relationships, and the role of personality traits and interpersonal identity processes in the subsistence of intimate relationships.

\section{Personality Traits and the Emergence of Relationships}

Personality traits have been found to be good predictors of the likelihood of finding a partner. Specifically, previous studies have found that the less neurotic and the more conscientious and extraverted individuals were, the more successful they were in finding an intimate partner (Neyer and Asendorpf 2001; Neyer and Lehnart 2007). In addition, individuals who found a partner exhibited decreases in Neuroticism-related traits (Lehnart et al. 2010; Neyer and Asendorpf 2001; Neyer and Lehnart 2007). Especially the latter finding shows that obtaining a full understanding of intimate relationships is important, as Neuroticism is associated strongly with psychopathology symptoms (Kotov et al. 2010).
Gender differences in how personality traits may contribute to finding a partner have rarely been assessed. However, Berry and Miller (2001) showed that Extraversion was associated negatively with the quality of social interactions in men, but not in women. Thus, Extraversion might be more important for men than for women in dating. Yet, studies on gender differences in the role of personality traits in becoming involved in serious intimate relationships are scarce.

Personality and the Course of Relationships: The Importance of Interpersonal Identity

Once individuals have a relationship, they will more or less identify themselves with this relationship. From a social investment perspective (Lodi-Smith and Roberts 2007), especially this identification is thought to drive personality maturation. Identification with choices in important life domains, such as intimate relationships, is a key aspect of identity formation (Erikson 1950). Marcia (1966) was among the first to examine empirically identity formation as described by Erikson (1950). He distinguished processes of commitment (i.e., identification with a certain option within a life domain) and exploration (i.e., examining different alternatives in a life domain). However, contemporary work points to the multi-faceted nature of exploration (Crocetti et al. 2008; Luyckx et al. 2006). First, individuals may reflect and gather information on the merits of their current commitments (Bosma 1985; Grotevant 1987; Meeus 1996). This process is referred to as in-depth exploration (Crocetti et al. 2008; Luyckx et al. 2006). Second, current commitments may be reevaluated and compared with alternatives. This process is referred to as reconsideration. Thus, to capture identity formation, one should consider processes of commitment, in-depth exploration, and reconsideration.

Identity formation takes place in several domains, including the intimate relationship domain (Erikson 1950; Grotevant et al. 1982). In this domain, commitment refers to deriving a sense of certainty in life from this relationship, in-depth exploration refers to reflecting on the relationship without necessarily questioning it, and reconsideration refers to having doubts about the relationship and considering alternative partners. Although the intimate relational domain is considered to be important (e.g., Bosma 1985; Grotevant et al. 1982; Meeus et al. 2007), it is relatively understudied.

A key aspect of intimate relational identity formation, interpersonal commitment, has been included in studies on relationship quality. In one such study, interpersonal commitment was related positively to Conscientiousness (Engel et al. 2002). Another study (Ahmetoglu et al. 2010) replicated these findings and also found a positive 
association between interpersonal commitment and Agreeableness. Thus, Conscientiousness and Agreeableness generally seem to be the most relevant correlates of interpersonal commitment. In both studies, there was little evidence for gender differences in the associations between personality traits and interpersonal commitment.

Overall, the aforementioned studies are characterized by a number of limitations. First, they are all cross-sectional and thereby not suited for inferring directionality in associations. Second, interpersonal exploration was not measured. Thus, the linkages between interpersonal identity processes and personality traits still need to be better examined.

Personality, Interpersonal Identity, and the Breakup of Relationships

If identification with social roles is indeed well-captured by identity processes, as the advocates of the social investment principle assume (e.g., Lodi-Smith and Roberts 2007), then one might expect a stronger interpersonal identity to be related to a willingness to proceed with a relationship. Hence, a strong interpersonal identity is likely to be associated with a lower probability of experiencing a breakup. In fact, because these identity processes should then be direct indicators of investment in a relationship, they should predict relationship stability (i.e., whether or not breakups will occur) above and beyond the effects of stable dispositions like personality traits.

Research relating personality traits to breakups mainly has been focused on marital relationships. An overview of these studies (Roberts et al. 2007) suggests that high Neuroticism, low Agreeableness, and low Conscientiousness are the most important predictors of relational breakups. However, the dissolution of non-marital relationships also can have detrimental effects, as it predicts suicide attempts in some individuals (Donald et al. 2006). Therefore, finding predictors of such breakups is important. Personality traits are not significant predictors in nonmarital relationships (Le et al. 2010; Neyer and Asendorpf 2001; Neyer and Lehnart 2007).

However, identity processes may predict relational breakups in non-marital intimate relationships. Specifically, previous studies suggest that individuals with higher levels of commitment, irrespective of their gender, are far less likely to face relational breakups (Le et al. 2010). Furthermore, Ahmetoglu et al. (2010) found that interpersonal commitment was a strong predictor of relational stability (i.e., relationship length), whereas Big Five personality traits had limited predictive power in this regard. Again, these findings applied to men and women. However, these studies were cross-sectional and focused on commitment while omitting other interpersonal identity processes. Thus, the unique contributions of personality traits and interpersonal identity processes in predicting the dissolution of intimate relationships still need to be examined.

\section{The Present Research}

The present research was guided by the social investment perspective, which holds that taking up and committing to social roles of adult life (e.g., a stable intimate relationship) is associated with changes in Big Five personality traits. To test whether this was the case, we employed a two-sample design that allowed us to distinguish replicable findings from incidental findings. Specifically, we pursued three more specific research goals with longitudinal data on a sample of female college students $(N=424$; Sample 1$)$ and a late adolescent community sample $(N=390$; Sample 2).

First, we examined whether personality traits were associated with the emergence of relationships. Based on previous research (Lehnart et al. 2010; Neyer and Asendorpf 2001; Neyer and Lehnart 2007), we especially expected a positive role for Extraversion in predicting the emergence of relationships, but more so for boys than for girls (Berry and Miller 2001).

Second, longitudinal associations of interpersonal identity processes with Big Five personality traits were examined. Based on previous research, interpersonal commitment was expected to be associated with Agreeableness and Conscientiousness with no substantial gender differences in these associations (Ahmetoglu et al. 2010; Engel et al. 2002). From a social investment perspective (e.g., Lodi-Smith and Roberts 2007), intimate relational identity processes also can be expected to be related negatively with Neuroticism. Guided by this perspective, we further expected interpersonal identity processes to predict changes in personality traits, with less evidence for predictive paths in the opposite direction. Because the present study was the first to include interpersonal in-depth exploration and reconsideration, strong hypotheses with regard to these processes cannot be advanced. However, reconsideration may represent a force opposing the certainty provided by commitments (e.g., Klimstra et al. 2010). Therefore, the role of this dimension may be inverse to the role of commitment. Unfortunately, reconsideration was only measured in our community sample.

Our third research goal was to provide a first longitudinal examination of what the best predictors of relational breakups were: personality traits or interpersonal identity processes. In line with previous studies (Ahmetoglu et al. 2010; Le et al. 2010; Neyer and Asendorpf 2001; Neyer and Lehnart 2007) and the social investment perspective, 
identity processes (especially commitment) were expected to be the most important predictors in this regard, for both boys and girls. The present study was the first to examine whether interpersonal exploration processes predicted relational breakups. Especially reconsideration as an indicator of uncertainty might be important in this regard, but it should again be noted that this dimension was only measured in our community sample.

\section{Method}

\section{Participants and Procedure}

Sample 1 consisted of participants drawn from the Leuven Trajectories of Identity Development Study (L-TIDES; Luyckx et al. 2006), a 7-wave longitudinal study on college students from the faculty of Psychology and Educational Sciences at a large Belgian university. Permission to undertake this study was granted by the Institutional Review Board within the researchers' department. Participants signed a standard consent form before participation and were informed that they could refuse or discontinue participation at any time. The first wave was collected at the end of 2002, and data on the Big Five of personality, interpersonal identity formation, and relationship status were available for four measurement occasions. Consequently, these four waves were used for the present study. There was a 1-year interval between each of these four measurement occasions. At Wave 1, a total of 565 individuals participated. Only individuals who participated in at least two out of four measurement waves $(N=485 ; 87.4 \%$ female $)$ were included. Because of the few boys in the sample, we decided to focus on girls only $\left(N=424 ; M_{\text {age }}\right.$ at Wave $1=18.6$ years, $\mathrm{SD}=0.57)$. Of these 424 girls, $83.5 \%$ came from intact families, $13.0 \%$ had divorced parents, whereas the other $3.5 \%$ reported a different family situation (e.g., one parent deceased).

Sample 2 comprised 390 late adolescents $(56.7 \%$ girls; $M_{\text {age }}=16.7$ years; $\mathrm{SD}=.80$ at Wave 1 ) participating in an on-going longitudinal research project on Conflict And Management Of Relationships (CONAMORE; Meeus et al. 2006). Participants were recruited from various high schools in the province of Utrecht, The Netherlands. Participants and their parents received an invitation letter, describing the research project and goals, and explaining the possibility to decline from participation. More than $99 \%$ of the approached adolescents decided to participate, and signed the informed consent form. Questionnaires were completed at the participants' own high school, during annual assessments in which all the study variables were included. Confidentiality of responses was guaranteed, verbal and written instructions were offered. Adolescents received $€ 10$ (approximately US \$13) as a reward for every wave in which they participated. This sample was assessed during five consecutive annual measurement waves, which resulted in a total age range from 16 to 20 years. Representative data on personality, identity, and intimate relationships was only available on the final two waves. That is, there were very few participants who had a relationship and filled out questionnaires on relational identity on most of the measurement waves, except for the last two measurement waves. Especially the size of the group of boys, which is already quite small now, was problematic at previous waves. Therefore, we decided to focus on the last two waves. In the remainder of the present manuscript, these two waves will be referred to as Wave 1 and 2, respectively. On the first of these waves, adolescents were 19.7 years $(\mathrm{SD}=.80)$ on average. Of these adolescents, $82.1 \%$ came from intact families, $15.5 \%$ had divorced parents, whereas the other $2.4 \%$ reported a different family situation.

In both Samples 1 and 2, there was no evidence for attrition-related biases, as Little's MCAR Test (Little 1988) revealed normed Chi squares $\left(\chi^{2} / \mathrm{df}\right)$ of 1.09 and 1.72 , respectively. According to guidelines by Bollen (1989), these values indicate that missing-data imputation is justified. In Sample 1 and 2, a multivariate analysis of variance on Wave 1 data yielded no significant multivariate effects of family situation on personality traits and identity processes. In addition, Chi square tests showed that family situation also was not associated with relationship status in the two samples. Age only was significantly associated with Openness in Sample 1 (i.e., $r=.14 ; p=.004$ ), and only with in-depth exploration in Sample 2 (i.e., $r=.17$; $p=.036$ ). Given that there were no consistent effects of demographic variables on our study variables, we did not control for demographic variables in subsequent analyses.

\section{Measures}

\section{Personality traits}

Sample 1 completed the Dutch version of the well-established 60-item NEO Five-Factor Inventory (NEO-FFI; Hoekstra et al. 1996) as a measure of Costa and McCrae's (1992) Five-Factor Model of personality. Sample items, as well as a detailed report on the psychometric properties, can be found in the NEO-FFI manual (Costa and McCrae 1992). In the present study, Cronbach's alphas across waves were $.87-.89$ for Neuroticism, $.80-.81$ for Extraversion, .69-.76 for Openness to Experience, .65-.70 for Agreeableness, and .78-.83 for Conscientiousness.

Sample 2 completed the Quick Big Five questionnaire (Goldberg 1992; Vermulst and Gerris 2005). In this 
instrument, a 7-point Likert scale, with a response format ranging from 1 (completely untrue) to 7 (completely true), is used to assess the Big Five personality dimensions. All dimensions are measured with 6 items each, such as: "talkative" (Extraversion), "sympathetic" (Agreeableness), "systematic" (Conscientiousness), "worried" (Neuroticism), and "creative" (Openness to Experience). For details concerning the psychometric properties of this questionnaire, the reader is referred to the Dutch manual (Vermulst and Gerris 2005). In the current study, reliability was high across waves for Extraversion (Cronbach's alphas .88-.91 for boys, and .89-.91 for girls), Agreeableness (Cronbach's alphas .83-.84 for boys, and .81-.83 for girls), Conscientiousness (Cronbach's alphas .89-.92 for boys, and .93 (twice) for girls), Neuroticism (Cronbach's alphas .83-.85 for boys, and .83-.84 for girls), and Openness to Experience (Cronbach's alphas .76-.81 for boys, and $.76-.77$ for girls).

\section{Interpersonal identity}

In Sample 1, we used the Utrecht-Groningen Identity Development Scale (Meeus 1996; Meeus and Dekovic 1995), a 10-item measure originally developed for use with Dutch-speaking adolescents to assess commitment (5 items) and in-depth exploration (5 items). Adolescents only completed this measure if they had a relationship. All items were answered on a 5-point Likert-type rating scale, ranging from 1 (strongly disagree) to 5 (strongly agree). Sample items are "My partner gives me certainty in life" (commitment), and "I try to find out a lot about my partner" (in-depth exploration). Meeus et al. (2002) provide an overview of the instrument's concurrent and construct validity, whereas evidence regarding the factorial structure is provided by Meeus (1996) and Meeus and Dekovic (1995). In the present study, Cronbach's alphas ranged from .87 to .90 for commitment, and from .50 to .69 for in-depth exploration.

In Sample 2, identity formation was assessed with the Utrecht-Management of Identity Commitments Scale (U-MICS), a self-report measure (Crocetti et al. 2008) based on the U-GIDS that was used in Sample 1. In addition to commitment and in-depth exploration scales, a reconsideration subscale (3 items; e.g., "I often think it would be better to try and find a different partner") is also distinguished in the U-MICS. A detailed account of its psychometric properties appears in Crocetti et al. (2008), and Crocetti et al. (2008). In the present study, reliability was acceptable for commitment (Cronbach's alphas .84-.90 for boys, .93-.94 for girls), in-depth exploration (Cronbach's alphas .62-.74 for boys, .74-.76 for girls), and reconsideration (Cronbach's alphas .84-.92 for boys, $.89-.90$ for girls).

\section{Relational Status}

Participants provided information on their relational status on each of the measurement waves, and also indicated how long they had been in their current relationship. We treated individuals who were involved in short-term dating relationships (relationships that lasted for less than 3 months) as not being involved in an intimate relationship, to be sure that we focused on more serious intimate relationships. For Sample 1, this information indicated that 36.6, 42.5, 52.6, and $43.4 \%$ of this all-girl sample had a relationship at Waves 1 through 4, respectively. The mean length of these relationships was $7.9,21.4,25.7$, and 33.2 months on the respective waves.

In Sample 2, 29.4, and $51.6 \%$ of the girls had a relationship on Waves 1 and 2, respectively. These figures were 12.4 and $27.2 \%$ for boys on Waves 1 and 2, respectively. With regard to relationship length, a categorical variable was used in Sample 2. This variable indicated that at Wave 1 , the frequencies of participants in the respective categories of "longer than 3 years", "between 1 and 3 years", "between 3 and 12 months" were 15.1, 49.6, and 35.3\%, respectively. At Wave 2, these figures were $26.9,43.1$, and $30 \%$, respectively. This information was further used to determine whether participants had a new relationship, still had the same relationship as on the previous measurement wave, or experienced a relational breakup between two consecutive waves.

\section{Results}

Personality Traits and Intimate Relationships

Our first goal was to assess whether personality traits predicted who would have intimate relationships. For this purpose, we employed logistic regression analyses. Big Five personality traits assessed at Wave 1 in the respective samples were predictors, with overall relationship status (i.e., indicating whether participants had no serious relationship (Sample 1: 175 girls; Sample 2: 99 girls and 115 boys) or at least one relationship of more than 3 months during the present study (Sample 1: 249 girls; Sample 2: 122 girls and 54 boys) as dependent variable. Contrary to our expectations, none of the Big Five traits significantly predicted who got involved in a relationship in Sample 1. In Sample 2, we ran separate analyses for boys and girls. Girls with higher levels of Extraversion were more likely to become involved in a relationship (Odds Ratio $=1.356$; $95 \% \mathrm{CI}=1.027,1.790 ; p=.032$ ). As hypothesized, boys with higher levels of Extraversion (Odds Ratio = 1.735; $95 \% \mathrm{CI}=1.148,2.624 ; p=.009$ ) were also more likely to become involved in a relationship, but the same was true 
for boys with higher levels of Neuroticism (Odds Ratio $=1.559 ; 95 \% \mathrm{CI}=1.043,2.329 ; p=.030$ ).

Associations Between Personality Traits and Identity Processes in Intimate Relationships

The second study goal was to examine how personality traits and interpersonal identity processes affected one another for individuals engaged in an intimate relationship, by means of cross-lagged panel models in Mplus 4.2 (Muthén and Muthén 2007). Such models contain stability paths (e.g., a regression path from $\mathrm{T} 1$ commitment to $\mathrm{T} 2$ commitment), initial correlations (e.g., correlations of $\mathrm{T} 1$ commitment with T1 Agreeableness), correlated change (e.g., correlations of $\mathrm{T} 2$ residuals of commitment with $\mathrm{T} 2$ residuals of Agreeableness; e.g., Neyer and Asendorpf 2001), and cross-paths (e.g., a regression path from T1 commitment to T2 Agreeableness). Model fit was judged by assessing the Root Mean Square Error of Approximation (RMSEA) and the Comparative Fit Index (CFI). RMSEAs below .08 and CFIs over .90 indicate adequate model fit (Kline, 2005).

We ran 10 models in Sample 1, with each of the models containing one Big Five trait and one identity dimension. For Sample 2, we ran 15 models, because each of the Big Five traits also was related to the new reconsideration dimension. These were multigroup models with boys and girls as groups. In all models, observed variables were used.

We focused on the individuals (Sample 1: 249 girls; Sample 2: 139 girls and 75 boys) who reported having at least one relationship that lasted more than 3 months during the present study. For these individuals, missing data were estimated by means of the Full Information Maximum Likelihood (FIML) procedure in Mplus. This was justified, because Little's (1988) MCAR Tests were significant $(p=.035$ and $p<.001$; in Samples 1 and 2, respectively), but normed Chi squares $\left(\chi^{2} / \mathrm{df}=1.14\right.$ and $\chi^{2} / \mathrm{df}=1.86$; in Samples 1 and 2 , respectively) were still acceptable according to guidelines by Bollen (1989).

To keep the models as parsimonious as possible, we attempted to constrain stability paths, correlated change, and cross-paths to be time-invariant in Sample 1. For Sample 2, adding time invariance constraints was not possible, because only two measurement waves were included. To examine whether adding time-invariance constraints in Sample 1 was justified, we compared the fit of models in which time-invariance constraints were added (i.e., constrained models) to models in which such constraints were not added (i.e., unconstrained models). Adding constraints was deemed justified if at least two out of the following three criteria were satisfied: (1) Chi square difference tests (Satorra and Bentler 2001) had to be non- significant, (2) differences in CFI should not exceed .010 (Cheung and Rensvold 2002), and (3) differences in RMSEA should not exceed .015 (Chen 2007). These criteria indicated that constraining stability paths was not justified in any model, constraining correlated change was justified in all but two models (i.e., the Extraversionexploration model and the Conscientiousness-exploration model), and constraining cross-paths was justified in all models. Therefore, standardized estimates for stability paths were not constrained to be time-invariant in any of the models, standardized estimates for cross-paths were constrained to be time-invariant in all models, and standardized estimates for correlated change were constrained to be time-invariant in 8 of the 10 models.

For Sample 2, we used a similar procedure to examine gender differences in correlated change and cross-paths. This procedure revealed gender differences in cross-paths in 1 of the 15 models (i.e., the model of commitment and Agreeableness), but no gender differences in correlated change in any of the models. Therefore, standardized estimates for correlated change were constrained to be equal for boys and girls in all models, whereas standardized estimates for cross-paths were constrained to be genderinvariant in all but 1 model.

Initial correlations between interpersonal identity processes and personality traits are presented in Table 1 . However, the associations of interest to the present study were correlated change associations and cross-lagged paths. Standardized estimates concerning these

Table 1 Time 1 correlations between personality traits and interpersonal identity processes in late adolescent female college students (Sample 1) and girls and boys from a community sample (Sample 2)

\begin{tabular}{lcclll}
\hline Dimension & $\mathrm{N}$ & $\mathrm{E}$ & $\mathrm{O}$ & $\mathrm{A}$ & $\mathrm{C}$ \\
\hline Sample 1: Girls & & & & & \\
$\quad(N=249)$ & & & & & \\
$\quad$ Commitment & $-.27^{* *}$ & $.23^{* *}$ & .02 & $.29^{* * *}$ & $.26^{* *}$ \\
$\quad$ In-depth exploration & .08 & .12 & .03 & .12 & $.21^{*}$ \\
Sample 2: Girls & & & & & \\
$\quad(N=122)$ & & & & & \\
Commitment & -.09 & -.06 & .09 & .10 & $.27^{* *}$ \\
In-depth exploration & -.13 & .00 & .12 & .04 & .05 \\
$\quad$ Reconsideration & -.14 & -.11 & .00 & .02 & -.09 \\
Sample 2: Boys & & & & & \\
$\quad(N=54)$ & & & & & .10 \\
Commitment & -.15 & -.01 & .13 & .05 & .10 \\
In-depth exploration & -.03 & .08 & .17 & .20 & .15 \\
Reconsideration & .26 & .06 & $.34 *$ & $.27^{*}$ & .21 \\
\hline
\end{tabular}

$N$ neuroticism, $E$ extraversion, $O$ openness, $A$ agreeableness, and $C$ conscientiousness

$* p<.05 ; * * p<.01 ; * * * p<.001$ 
Table 2 Correlated change and cross-lagged associations between interpersonal identity and personality in late adolescent college student girls

\begin{tabular}{|c|c|c|c|c|c|c|}
\hline \multirow[t]{2}{*}{ Associations } & \multicolumn{3}{|c|}{ Commitment } & \multicolumn{3}{|c|}{ Exploration } \\
\hline & $\mathrm{T} 2$ & $\mathrm{~T} 3$ & $\mathrm{~T} 4$ & $\mathrm{~T} 2$ & T3 & $\mathrm{T} 4$ \\
\hline \multicolumn{7}{|l|}{ Correlated change } \\
\hline Neuroticism & $-.07 * *$ & $-.06 * *$ & $-.07 * *$ & .01 & .01 & .01 \\
\hline Extraversion & .02 & .02 & .02 & .00 & $.13 * *$ & -.02 \\
\hline Openness & -.02 & -.02 & -.02 & .04 & .03 & .03 \\
\hline Agreeableness & $.06^{*}$ & $.06^{*}$ & $.06^{*}$ & $.08 * *$ & $.07 * *$ & $.07 * *$ \\
\hline \multirow[t]{2}{*}{ Conscientiousness } & $.05^{*}$ & $.05^{*}$ & $.05^{*}$ & .01 & .05 & $-.13 * *$ \\
\hline & $\mathrm{T} 1-\mathrm{T} 2$ & $\mathrm{~T} 2-\mathrm{T} 3$ & T3-T4 & $\mathrm{T} 1-\mathrm{T} 2$ & T2-T3 & $\mathrm{T} 3-\mathrm{T} 4$ \\
\hline \multicolumn{7}{|l|}{ Identity $\rightarrow$ personality } \\
\hline Neuroticism & -.02 & -.01 & -.02 & .02 & .02 & .02 \\
\hline Extraversion & .05 & .05 & .06 & .01 & .01 & .01 \\
\hline Openness & $.05^{*}$ & $.05^{*}$ & $.06^{*}$ & .01 & .01 & .02 \\
\hline Agreeableness & .03 & .03 & .03 & -.03 & -.03 & -.04 \\
\hline Conscientiousness & $.12 * * *$ & $.11 * * *$ & $.13 * * *$ & -.02 & -.02 & -.03 \\
\hline \multicolumn{7}{|l|}{ Personality $\rightarrow$ identity } \\
\hline Neuroticism & $-.10 * *$ & $-.09 * *$ & $-.10 * *$ & .03 & .02 & .02 \\
\hline Extraversion & $.09 *$ & $.09 *$ & $.08^{*}$ & -.03 & -.03 & -.03 \\
\hline Openness & .04 & .04 & .04 & -.01 & -.01 & -.01 \\
\hline Agreeableness & .04 & .04 & .03 & -.04 & -.03 & -.03 \\
\hline Conscientiousness & .06 & .06 & .06 & $.07 *$ & $.07 *$ & $.06 *$ \\
\hline
\end{tabular}

associations and paths appear in Table 2 for Sample 1, and in Table 3 for Sample 2.

Table 2 reveals correlated changes between interpersonal identity processes and personality traits. Somewhat unexpectedly, changes in interpersonal commitment were correlated negatively with changes in Neuroticism. However, in line with our hypotheses, changes in interpersonal commitment were correlated positively with changes in Agreeableness and Conscientiousness. Changes in interpersonal in-depth exploration were correlated positively with changes in Agreeableness. There was some evidence for positive correlated change between interpersonal in-depth exploration and Extraversion, and negative correlated change between interpersonal in-depth exploration and Conscientiousness. However, these associations were only significant on one of the three measurement occasions for correlated change.

Table 3 shows that there was less evidence for correlated change between personality traits and interpersonal identity processes in Sample 2. There was only significant negative correlated change between Conscientiousness and interpersonal reconsideration, indicating that relative increases in reconsideration were associated with relative decreases in Conscientiousness in both boys and girls. This finding was in line with our hypotheses.

In Sample 1, interpersonal identity processes predicted relative changes in two personality traits (see Table 2). Specifically, interpersonal commitment unexpectedly predicted relative increases in Openness, but, as hypothesized, also in Conscientiousness. Interpersonal in-depth exploration did not predict personality traits. Findings in Sample 2 confirmed our hypotheses, as interpersonal identity processes predicted relative changes in the personality trait of Agreeableness (see Table 3). That is, interpersonal commitment predicted relative increases in Agreeableness in boys, but not in girls. Interpersonal reconsideration predicted relative decreases in Agreeableness for both boys and girls.

There were also predictive paths from personality traits to interpersonal identity processes. Unexpectedly, our findings in Sample 1 suggest that Neuroticism predicted relative decreases in interpersonal commitment, whereas Extraversion predicted relative increases in this identity dimension (see Table 2). Conscientiousness predicted relative increases in interpersonal in-depth exploration. In Sample 2, there was only one significant path, indicating that Neuroticism predicted relative increases in interpersonal reconsideration in both boys and girls (see Table 3). This finding was not anticipated.

Personality Traits and Interpersonal Identity Processes as Predictors of Relational Stability

Our third study goal was to examine whether personality traits and interpersonal identity processes could predict who, among those involved in a relationship, would be the 
Table 3 Correlated change and cross-lagged associations between interpersonal identity and personality in late adolescent boys and girls from a community sample

\begin{tabular}{|c|c|c|c|c|c|c|}
\hline \multirow{2}{*}{ Associations } & \multicolumn{3}{|l|}{ Boys } & \multicolumn{3}{|l|}{ Girls } \\
\hline & $\mathrm{C}$ & $\mathrm{E}$ & $\mathrm{R}$ & $\mathrm{C}$ & $\mathrm{E}$ & $\mathrm{R}$ \\
\hline \multicolumn{7}{|l|}{ Correlated change } \\
\hline Neuroticism & .02 & .06 & .00 & .02 & .07 & .00 \\
\hline Extraversion & .02 & -.02 & -.01 & .01 & -.02 & -.01 \\
\hline Openness & -.06 & .02 & .05 & -.05 & .02 & .04 \\
\hline Agreeableness & .10 & .04 & -.01 & .09 & .04 & -.01 \\
\hline Conscientiousness & .07 & .02 & $-.10 *$ & .06 & .02 & $-.10^{*}$ \\
\hline \multicolumn{7}{|c|}{ Identity $\rightarrow$ personality } \\
\hline Neuroticism & .06 & .00 & .03 & .08 & .00 & .04 \\
\hline Extraversion & -.03 & .00 & -.05 & -.04 & .01 & -.05 \\
\hline Openness & .06 & .02 & -.11 & .07 & .03 & -.09 \\
\hline Agreeableness & $.38 * * *$ & .04 & $-.15^{*}$ & .10 & .06 & $-.17 *$ \\
\hline Conscientiousness & .06 & -.01 & -.04 & .07 & -.02 & -.04 \\
\hline \multicolumn{7}{|l|}{ Personality $\rightarrow$ identity } \\
\hline Neuroticism & -.07 & -.05 & $.18^{*}$ & -.06 & -.05 & $.17 *$ \\
\hline Extraversion & .06 & .00 & -.12 & .06 & .00 & -.13 \\
\hline Openness & .12 & .07 & .08 & .09 & .06 & .07 \\
\hline Agreeableness & -.05 & .02 & -.10 & .15 & .02 & -.08 \\
\hline Conscientiousness & -.04 & -.10 & -.05 & -.03 & -.10 & -.05 \\
\hline
\end{tabular}

most likely to face a breakup. For this purpose, logistic regression was employed. We checked at each measurement wave whether participants' relationships had survived across the interval between that measurement wave and the next. Using this information, a dichotomous variable (for Sample 1 measured at Waves 2, 3, and 4, respectively; for Sample 2 measured at Wave 2) was created, which served as dependent variable in our model. Interpersonal identity processes and personality traits at the preceding measurements (i.e., for Sample 1: Waves 1, 2, and 3, respectively; for Sample 2: Wave 1) were used as independent variables. Data were reordered in such a way that personality and identity variables at the wave preceding the breakup served as predictors. Each case was considered only once to avoid statistical dependency effects. Specifically, we only considered the first measurement occasion for individuals who did not experience any breakup, and only considered the first breakup for the other individuals. It should be noted that a logistic regression in which cases were entered multiple times (i.e., with no control for dependency) yielded highly similar results.

In Sample 1, 58 of the participants who reported being in at least one relationship $(23.3 \%)$ experienced at least one breakup. Variables predicting these breakups were entered in two steps. In Step 1, personality traits were entered, whereas identity processes were entered in Step 2 to examine whether these processes could predict relational breakups above and beyond personality traits. When only personality traits were considered in Step 1, no significant effects were found. In line with our hypotheses, interpersonal identity processes (i.e., Step 2) did significantly add to the prediction of relational breakups. That is, individuals with lower levels of commitment (Odds Ratio $=.285$; $95 \% \mathrm{CI}=.163, .498 ; p<.001)$ were more likely to face relational breakups.

In Sample 2, only 14 of the boys (25.9\%) and 12 of the girls $(9.8 \%)$ who were involved in a relationship experienced a breakup. Therefore, we ran a model in which we controlled for sex by including it in the first step of our model. Sex was a significant predictor in Step 1 (Odds Ratio $=4.210 ; 95 \% \mathrm{CI}=1.714,10.343 ; p=.002)$, with boys being more likely to face breakups than girls. After adding the personality traits (i.e., Step 2), sex was still a significant predictor (Odds Ratio $=3.999 ; \quad 95 \%$ $\mathrm{CI}=1.512,10.573 ; p=.005)$. Extraversion was also a significant predictor (Odds Ratio $=1.868 ; \quad 95 \%$ $\mathrm{CI}=1.001,3.485 ; p=.049)$, as individuals with higher levels of Extraversion were more likely to face breakups. Sex (Odds Ratio $=3.491 ; 95 \%$ CI $=1.281$, 9.512; $p=.014$ ) and Extraversion (Odds Ratio $=1.987 ; 95 \%$ $\mathrm{CI}=1.022,3.862 ; p=.043)$ remained significant predictors when the interpersonal identity processes were considered (i.e., Step 3). In addition, our hypothesis was again confirmed as these processes added significantly to the prediction of relational breakups. Specifically, individuals with higher levels of reconsideration were more 
likely to face a relational breakup (Odds Ratio $=2.496$; $95 \% \mathrm{CI}=1.123,5.546 ; p=.025)$.

\section{Discussion}

In the period from late adolescence through young adulthood, individuals are expected to invest gradually in social roles of adult life, such as establishing a stable relationship. From a social investment perspective (e.g., Lodi-Smith and Roberts 2007), investment in such roles is thought to drive personality maturation. To partly test this principle, the present study provides a first longitudinal examination of the associations between personality traits, interpersonal identity processes and intimate relationships. Two samples were employed to check whether findings were replicable. We first showed that specific personality traits (i.e., especially Extraversion) predicted who would become involved in a relationship, but more so for boys than for girls. Second, the social investment principle was supported in the sense that personality traits were associated with interpersonal identity processes. Finally, we showed that interpersonal identity processes were the best predictors of relational breakups. Collectively, these findings advanced our knowledge on the role of personality and identity processes in the establishment of stable intimate relationships.

Personality Traits and the Emergence of Intimate Relationships

Our findings suggest that personality traits may determine partly who will become engaged in an intimate relationship. That is, in line with previous studies (Lehnart et al. 2010; Neyer and Asendorpf 2001; Neyer and Lehnart 2007), Extraversion was found to be the best predictor of whether individuals would have a relationship during the time span covered in our study. However, this finding mainly applied to boys. The association with Extraversion was weaker for girls in the community sample, and nonsignificant in female college students.

Previous studies already suggested that personality traits may play a smaller role in the emergence of intimate relationships for girls than for boys. For example, an observational study (Berry and Miller 2001) demonstrated that low levels of Extraversion may lead boys, but not girls, to be less competent in interacting with potential dating partners. Previous studies (Lehnart et al. 2010; Neyer and Asendorpf 2001; Neyer and Lehnart 2007) showed that engaging in interpersonal relationships is associated with decreases in Neuroticism (i.e., the personality trait most strongly related to symptoms of psychopathology; Kotov et al. 2010), which underscores the potential importance of these relationships. Hence, interventions specifically focusing on the social interaction skills of introverted boys may be useful.

In light of these previous studies, our finding that boys with higher levels of Neuroticism were more likely to be involved in an intimate relationship was rather counterintuitive. However, one of these studies (Neyer and Lehnart 2007) had similar results, as singles with higher levels of Neuroticism were more likely to get a relationship. In that study, it was speculated that this may be due to positive side effects of Neuroticism, such as having a stronger need and a stronger motivation for engaging in social relationships.

Associations Between Personality Traits and Interpersonal Identity Processes

A main premise of the social investment model is that commitment to social roles of adult life should be associated with changes in personality traits. Therefore, our second goal was to provide a first examination of the longitudinal linkages of personality traits with interpersonal identity processes. Ahmetoglu et al. (2010) previously showed that Agreeableness tends to be associated with a sense of security derived from intimate relationships (i.e., interpersonal commitment). We replicated, but also expanded on, these findings. That is, we found some evidence suggesting that higher levels of Agreeableness also may go together with thorough reflection (i.e., high levels of in-depth exploration) and few doubts concerning these relationships (i.e., lower levels of interpersonal reconsideration). However, these associations only were found in either the college sample, or the community sample. Furthermore, it is unclear whether Agreeableness predicts a strong interpersonal identity, or whether a strong interpersonal identity predicts Agreeableness. Still, high levels of Agreeableness clearly are associated with a strong interpersonal identity (i.e., high levels of commitment and in-depth exploration, and little reconsideration).

Similarly, in line with previous research (Ahmetoglu et al. 2010; Engel et al. 2002), relatively consistent associations of interpersonal identity with Conscientiousness were found. Specifically, there was positive correlated change of interpersonal commitment with Conscientiousness in the college sample, and negative correlated change of Conscientiousness with interpersonal reconsideration in the community sample. Collectively, these results suggest that individuals gain a stronger sense of interpersonal identity once they become more conscientious. Again, these findings confirmed those obtained in previous work.

In line with the social investment principle (e.g., LodiSmith and Roberts 2007), Neuroticism was also associated consistently with interpersonal identity. This trait predicted 
a weakened sense of interpersonal commitment in college students, and increased doubts (i.e., reconsideration) in the community sample. Previous studies specifically focusing on intimate relationships found little evidence for such associations, but Neuroticism is one of the most consistent predictors of problems in identity formation in other identity domains (e.g., Crocetti et al. 2008; Klimstra et al. 2012; Klimstra et al. in press). Thus, Neuroticism appears to be associated consistently with a strong identity, regardless of the domain that is considered.

Extraversion and Openness were not associated consistently with interpersonal identity, and in-depth exploration was not associated consistently with Big Five personality traits. That is, associations involving these variables that were found in the college sample were not replicated in the community sample. For in-depth exploration, this may be due to its complex nature. Theoretically, in-depth exploration should have a positive effect on identity formation, as it concerns the evaluation of one's existing commitments without necessarily questioning these commitments (Crocetti et al. 2008). However, continuous evaluation of one's commitments may amount to rumination. As a result, it has been shown that in-depth exploration is much associated more consistently with other variables once ruminative tendencies were controlled for (Luyckx et al. 2008). Thus, including and controlling for ruminative tendencies may provide more consistent associations of Big Five personality traits with interpersonal in-depth exploration.

Only Agreeableness, Conscientiousness, and Neuroticism, which are the three key traits of the social investment principle (e.g., Roberts and Wood 2006) are associated more or less consistently with interpersonal identity formation. In addition, only commitment is thought to be a key indicator of social investment (Lodi-Smith and Roberts 2007). Therefore, our finding that only interpersonal commitment and its opposing force (i.e., interpersonal reconsideration) were associated with these three key traits provides support for the social investment principle. A further key assumption of the social investment principle is that increased investment (i.e., increased commitment and decreased reconsideration) should predict changes in Neuroticism, Agreeableness, and Conscientiousness. We found only limited evidence for this proposed directionality. That is, Agreeableness was predicted by interpersonal commitment and reconsideration in the community sample only, and Conscientiousness was predicted by interpersonal commitment in college students only. Still, our findings provide some support for the social investment principle.

Both mature levels on Big Five personality traits (e.g., Kotov et al. 2010) and a strong sense of identity (e.g., Crocetti et al. 2008; Luyckx et al. 2008) have been shown to be associated with psychological well-being. Therefore, our findings do not just advance theorizing on the social investment principle. Instead, psychological health professionals working with young people also should take notice of the importance of identity processes and personality traits for positive psychosocial development in general.

Personality Traits, Interpersonal Identity, and Relational Stability

In line with the social investment principle, interpersonal identity processes were indeed better predictors of relational breakups than personality traits were. Specifically, in line with previous research (Ahmetoglu et al. 2010; Le et al. 2010; Neyer and Asendorpf 2001; Neyer and Lehnart 2007), our findings for the college student sample suggested that interpersonal commitment was the most relevant predictor of relational breakups. However, findings in our community sample suggested that reconsideration (which was not considered in our college student sample), not commitment, might be the superior predictor of relational breakups. Commitment represents psychological investment, whereas reconsideration reflects uncertainty with regard to this investment and consideration of different relationships to invest in (Crocetti et al. 2008; Klimstra et al. 2010). Our findings therefore suggest that, for the stability of intimate relationships, uncertainty might be even more detrimental than a lack of psychological investment.

The findings of our final study goal underscore the importance of examining interpersonal identity. This importance becomes even more significant if one considers how serious the implications of breakups of intimate relations can be for psychological well-being (e.g., Donald et al. 2006). These findings once more underscore the importance of positive identity formation, and may call for interventions for individuals who repeatedly fail to gradually identify themselves with a relationship.

\section{Strengths and Limitations}

The present research is characterized by several strengths. First, we employed data from two samples. Another strength was that both studies were longitudinal with largeenough samples to employ relatively sophisticated techniques such as cross-lagged panel models (Burkholder and Harlow 2003). This allowed us to examine the directionality in the associations between personality traits and identity processes. Finally, previous research on intimate relationships had included measures of interpersonal commitment, but did not include measures of exploration. Identity formation can only be measured appropriately if exploration measures also are included (Marcia 1966). Therefore, the present study expanded on previous research 
on intimate relationships through its reliance on identity measures that captured the complex, multidimensional nature of the construct.

Despite these strengths, there were also several limitations that need to be mentioned. The fact that we relied exclusively on self-reported data can be considered a first limitation. Identity formation is an internal process that is best captured by means of self-reports, but for personality assessment other-reports (e.g., peer-reports and parentreports) also are used widely. However, it has been shown that such other-reported data also are biased slightly (e.g., Branje et al. 2003).

Second, the reliability of our in-depth exploration scale was not optimal on all measurement occasions, as Cronbach's Alpha was .50 on one of the measurement occasions in Sample 1. These reliability issues appear to be solved in the new version of this scale (Crocetti et al. 2008) that was used in the community sample. Therefore, we encourage the use of the new version of the in-depth exploration scale in future studies.

Third, we had little information about the adolescents' partners. As a result, we do not know whether adolescents were involved in homosexual or heterosexual relationships, and what scores their partners had on personality traits and relational identity processes. Thus, the present research merely examined self-related processes with regard to intimate relationships.

Fourth, relatively few boys were included in the two samples. Therefore, we were only able to examine gender differences in Sample 2. Yet, the present study was still the first to explicitly test for gender differences in some of the research questions under investigation.

Fifth, we ran a large number of tests, which yielded a large number of null findings. This suggests that only a few specific personality traits and identity processes affect one another, and are involved in predicting the initiation and dissolution of intimate relationships. Alternatively, it could be argued that the relatively few significant results are due to chance. However, we countered this potential limitation by using a two-sample design that allowed us to distinguish replicable results from incidental findings.

A final potential limitation concerns the use of a phraseitem questionnaire (i.e., the NEO-FFI; Costa and McCrae 1992) in Sample 1 and an adjective-questionnaire (i.e., the Quick Big Five; Vermulst and Gerris 2005) in Sample 2. Briggs (1992) showed that such different kind of Big Five questionnaires were strongly, but not perfectly, correlated with one another. Thus, phrase items and adjective items may tap the same construct, but in slightly different ways. Although this may be one of the reasons why our results were not always replicated in both samples, the findings that were replicated may be particularly robust. For that reason, this final limitation may ultimately prove to be a strength.

\section{Conclusion}

Despite the aforementioned limitations, the present study contributes to the literature on social investment and the course of intimate relationships in several important ways. That is, our findings suggest that especially highly extraverted individuals are likely to become involved in a relationship. In the individuals who get involved in intimate relationships, an interpersonal identity evolves. In line with the social investment principle, especially individuals with low levels of Neuroticism and high levels of Agreeableness and Conscientiousness tend to have strong interpersonal identities. Although there was little consistency as regards the directionality in these longitudinal associations, knowledge on these linkages is still of utmost importance as identity processes were significant predictors of relational breakups. Personality traits only played a limited role in this regard. Overall, our study provides further insight into the role of personality and interpersonal identity in the emergence, maintenance, and subsistence of intimate relationships in late adolescence and young adulthood.

Acknowledgments TK conceived of the study, drafted the manuscript, and conducted the analyses; KL coordinated data collection of Sample 1 and helped in drafting the manuscript; SB participated in the design of the study from which Sample 2 data was drawn and helped in drafting the manuscript; ET helped in drafting the manuscript; LG designed the study from which the data for Sample 1 was drawn and helped drafting the manuscript; WM designed the study from which Sample 2 data was drawn and helped in drafting the manuscript.

\section{References}

Ahmetoglu, G., Swami, V., \& Chamorro-Premuzic, T. (2010). The relationship between dimensions of love, personality, and relationship length. Archives of Sexual Behavior, 39, 1181-1190.

Berry, D. S., \& Miller, K. M. (2001). When boy meets girl: Attractiveness and the Five-Factor Model in opposite-sex interactions. Journal of Research in Personality, 35, 62-77.

Bollen, K. (1989). Structural equations with latent variables. New York: Wiley.

Bosma, H. A. (1985). Identity development in adolescence: Coping with commitments. Unpublished doctoral dissertation, University of Groningen, The Netherlands.

Branje, S. J. T., van Aken, M. A. G., van Lieshout, C. F. N., \& Mathijssen, J. J. J. P. (2003). Personality judgments in adolescents' families: The perceiver, the target, their relationship, and the family. Journal of Personality, 71, 49-81.

Briggs, S. R. (1992). Assessing the Five-Factor Model of personality description. Journal of Personality, 60, 253-293.

Burkholder, G. J., \& Harlow, L. L. (2003). An illustration of a longitudinal cross-lagged design for larger structural equation models. Structural Equation Modeling, 10, 465-486. 
Chen, F. F. (2007). Sensitivity of goodness of fit indexes to lack of measurement invariance. Structural Equation Modeling, 14, 464-504.

Cheung, G. W., \& Rensvold, R. B. (2002). Evaluating goodness-of-fit indexes for testing measurement invariance. Structural Equation Modeling, 9, 233-255.

Costa, P. T., Jr, \& McCrae, R. R. (1992). Revised NEO personality inventory (NEO-PI-R) and the five factor inventory (NEO-FFI): Professional manual. Odessa, FL: Psychological Assessment Resources.

Crocetti, E., Rubini, M., Luyckx, K., \& Meeus, W. (2008a). Identity formation in early and middle adolescents from various ethnic groups: From three dimensions to five statuses. Journal of Youth and Adolescence, 37, 983-996.

Crocetti, E., Rubini, M., \& Meeus, W. H. J. (2008b). Capturing the dynamics of identity formation in various ethnic groups: Development and validation of a three-dimensional model. Journal of Adolescence, 31, 207-222.

Donald, M., Dower, J., Correa-Velez, I., \& Jones, M. (2006). Risk and protective factors for medically serious suicide attempts: A comparison of hospital-based with population-based samples of young adults. Australian and New Zealand Journal of Psychiatry, 40, 87-96.

Engel, G., Olson, K. R., \& Patrick, C. (2002). The personality of love: Fundamental motives and traits related to components of love. Personality and Individual Differences, 32, 839-853.

Erikson, E. H. (1950). Childhood and society. New York: Norton.

Goldberg, L. R. (1992). The development of markers for the Big-Five factor structure. Psychological Assessment, 4, 26-42.

Grotevant, H. D. (1987). Toward a process model of identity formation. Journal of Adolescent Research, 2, 203-222.

Grotevant, H. D., Thorbecke, W., \& Meyer, M. L. (1982). An extension of Marcia's identity status interview into the interpersonal domain. Journal of Youth and Adolescence, 11, 33-47.

Hoekstra, H. A., Ormel, J., \& De Fruyt, F. (1996). NEO persoonlijkheids vragenlijsten: NEO-PI-R, NEO-FFI. Handleiding [NEO personality questionnaires: NEO-PI-R and NEO-FFI-Manual]. Lisse, The Netherlands: Swets \& Zeitlinger.

Klimstra, T. A., Luyckx, K., Germeijs, V., Meeus, W. H. J., \& Goossens, L. (2012). Personality traits and educational identity formation in late adolescents: Longitudinal associations and academic progress. Journal of Youth and Adolescence, 41, 346-361.

Klimstra, T. A., Luyckx, K., Goossens, L., Teppers, E., \& De Fruyt, F. (in press). Associations of identity dimensions with Big Five personality domains and facets. European Journal of Personality. doi:10.1002/per.1853.

Klimstra, T. A., Luyckx, K., Hale, W. W., Frijns, T., van Lier, P. A. C., \& Meeus, W. H. J. (2010). Short-term fluctuations in identity: Introducing a micro-level approach to identity formation. Journal of Personality and Social Psychology, 99, 191-202.

Kline, R. B. (2005). Principles and practice of structural equation modeling (2nd ed.). New York: Guilford Press.

Kotov, R., Gamez, W., Schmidt, F., \& Watson, D. (2010). Linking "big" personality traits to anxiety, depressive, and substance use disorders: A meta-analysis. Psychological Bulletin, 136, 768-821.

Le, B., Dove, N. L., Agnew, C. R., Korn, M. S., \& Mutso, A. A. (2010). Predicting non-marital romantic relationship dissolution: A meta-analytic synthesis. Personal Relationships, 17, 377-390.

Lehnart, J., Neyer, F. J., \& Eccles, J. (2010). Long-term effects of social investment: The case of partnering in young adulthood. Journal of Personality, 78, 639-670.

Little, R. (1988). A test of missing completely at random for multivariate data with missing values. Journal of the American Statistical Association, 83, 1198-1202.
Lodi-Smith, J., \& Roberts, B. W. (2007). Social investment and personality: A meta-analysis of the relationship of personality traits to investment in work, family, religion, and volunteerism. Personality and Social Psychology Review, 11, 68-86.

Luyckx, K., Goossens, L., \& Soenens, B. (2006a). A developmentalcontextual perspective on identity construction in emerging adulthood: Change dynamics in commitment formation and commitment evaluation. Developmental Psychology, 42, 366-380.

Luyckx, K., Goossens, L., Soenens, B., \& Beyers, W. (2006b). Unpacking commitment and exploration: Preliminary validation of an integrative model of late adolescent identity formation. Journal of Adolescence, 29, 361-378.

Luyckx, K., Schwartz, S. J., Berzonsky, M. D., Soenens, B., Vansteenkiste, M., Smits, I., et al. (2008). Capturing ruminative exploration: Extending the four-dimensional model of identity formation in late adolescence. Journal of Research in Personality, 42, 58-82.

Marcia, J. E. (1966). Development and validation of ego-identity status. Journal of Personality and Social Psychology, 3, 551-558.

Meeus, W. H. J. (1996). Studies on identity development in adolescence: An overview of research and some new data. Journal of Youth and Adolescence, 25, 569-598.

Meeus, W. H. J., Akse, J., Branje, S. J. T., Ter Bogt, T. F. M., Crommelin, P. M., Delsing, M. J. M. H., et al. (2006). Codebook of the research project CONflict And Management Of RElationships (CONAMORE). Unpublished manuscript, Utrecht University, The Netherlands.

Meeus, W. H. J., Branje, S. J. T., van der Valk, I., \& de Wied, M. (2007). Relationships with intimate partner, best friend, and parents in adolescence and early adulthood: A study of the saliency of intimate partnership. International Journal of Behavioral Development, 31, 569-580.

Meeus, W., \& Dekovic, M. (1995). Identity development, parental and peer support in adolescence: Results of a national, Dutch survey. Adolescence, 30, 931-944.

Meeus, W., Oosterwegel, A., \& Vollebergh, W. (2002). Parental and peer attachment and identity development in adolescence. Journal of Adolescence, 25, 93-106.

Muthén, L. K., \& Muthén, B. O. (2007). Mplus user's guide (4th ed.). Los Angeles, CA: Muthén \& Muthén.

Neyer, F. J., \& Asendorpf, J. B. (2001). Personality-relationship transaction in young adulthood. Journal of Personality and Social Psychology, 81, 1190-1204.

Neyer, F. J., \& Lehnart, J. (2007). Relationships matter in personality development: Evidence from an 8-year longitudinal study across young adulthood. Journal of Personality, 75, 535-568.

Roberts, B. W., Kuncel, N. R., Shiner, R., Caspi, A., \& Goldberg, L. R. (2007). The power of personality: The comparative validity of personality traits, socioeconomic status, and cognitive ability for predicting important life outcomes. Perspectives on Psychological Science, 2, 313-345.

Roberts, B. W., Walton, K. E., \& Viechtbauer, W. (2006). Patterns of mean-level change in personality traits across the life course: A meta-analysis of longitudinal studies. Psychological Bulletin, $132,1-25$.

Roberts, B. W., \& Wood, D. (2006). Personality development in the context of the neo-Socioanalytic Model of personality. In D. Mroczek \& T. Little (Eds.), Handbook of personality development (pp. 11-39). Mahwah, NJ: Erlbaum.

Satorra, A., \& Bentler, P. M. (2001). A scaled difference Chi square test statistic for moment structure analysis. Psychometrika, 66, 507-514.

Vermulst, A. A., \& Gerris, J. R. M. (2005). QBF: Quick Big Five persoonlijkheidstest handleiding [Quick Big Five personality test manual]. Leeuwarden, The Netherlands: LDC Publications. 


\section{Author Biographies}

Theo A. Klimstra is Assistant Professor at Tilburg University, The Netherlands. His major research interests include personality, identity, and adolescent development.

Koen Luyckx is a Research Professor for the Fund of Scientific Research (FWO), Flanders, Belgium at the Catholic University Leuven, Belgium. His major research interests include identity, personality, parenting, and chronic illness.

Susan Branje is Professor at the Research Centre Adolescent Development of Utrecht University. Her research interests include development of personality, psychosocial adjustment, and relationships with parents and friends in adolescence.
Eveline Teppers is a $\mathrm{PhD}$ student in Developmental Psychology at the Catholic University of Leuven, Belgium. Her research interests include adolescent loneliness, personality, and social network use.

Luc Goossens is a Full Professor in Developmental Psychology at the Catholic University of Leuven, Belgium. His major research interests include adolescent identity, autonomy, and loneliness.

Wim H. J. Meeus is Full Professor of Adolescent Development and chair of the Research Centre Adolescent Development of Utrecht University, The Netherlands. His major research interests include personality, identity, and relationships in adolescence. 